\title{
Austriaca
}

Cahiers universitaires d'information sur l'Autriche

\section{„Das könnte man gesellschaftlich so sagen, aber es wäre weiter nichts als eine vertuschende Redeweise" (Theodor, 1. Akt, 12. Szene)}

Gesprächs- und emotionslinguistische Beobachtungen von Hofmannsthals Lustspiel Der Unbestechliche, Akt I

Monika Wolf, "Cela pourrait être dit de façon politiquement correcte, mais ce ne serait rien de plus qu'une dissimulation de la parole » (Theodor, acte I, sc. 1). Observations linguistiques conversationnelles et émotionnelles de la pièce de Hofmannsthal, The Incorruptible (acte I).

Monika Wolf, "This could be said socially, but it would be nothing more than a cover-up of speech" (Theodor, Act 1, Scene 1). Conversational and emotional linguistic observations of Hofmannsthal's play The Incorruptible, Act I

\section{Monika Wolf}

\section{(2) OpenEdition}

\section{Édition électronique}

URL : https://journals.openedition.org/austriaca/766

DOI : 10.4000 /austriaca.766

ISSN : 2729-0603

\section{Éditeur}

Presses universitaires de Rouen et du Havre

\section{Édition imprimée}

Date de publication : 1 décembre 2019

Pagination : 191-209

ISBN : 979-10-240-1454-8

ISSN : 0396-4590

Référence électronique

Monika Wolf, vertuschende Redeweise" (Theodor, 1. Akt, 12. Szene)", Austriaca [Online], 88-89|2019, Online erschienen am: 31 Dezember 2020, abgerufen am 24 Oktober 2022. URL: http:// journals.openedition.org/austriaca/766 ; DOI: https://doi.org/10.4000/austriaca.766 
Monika Wolf

Université Toulouse Jean-Jaurès

\section{„Das könnte man gesellschaftlich so sagen, aber es wäre weiter nichts als eine vertuschende Redeweise" (Theodor, 1. Akt, 12. Szene) Gesprächs- und emotionslinguistische Beobachtungen von Hofmannsthals Lustspiel Der Unbestechliche, Akt I}

Sprache vollzieht sich primär dialogisch und dient nicht nur dazu, Bewusstseinsinhalte, d.h. Kognitives oder Emotionales auszudrücken, sondern auch soziale Rollen in der Interaktion festzulegen. Im sozialen und individuellen Leben spielen Emotionen eine zentrale Rolle, ohne Emotionen kann Interaktion kaum stattfinden. Seinen Bewusstseinsinhalten durch die Sprache Ausdruck zu verleihen, ermöglicht dem Menschen, diese Inhalte kommunizierbar zu machen und so ein ausgeprägtes Sozialleben zu führen; dies gehört damit zu seinen wichtigsten Fähigkeiten. Der Mensch kommuniziert aber eher selten, um einfach nur seine Innenwelt zu offenbaren, sondern setzt seine Mitteilungen strategisch für einen bestimmten Zweck ein. Dabei kommt es auch vor, dass bestimmte Gefühle aus taktischen Gründen simuliert bzw. verborgen werden: „Eine wesentliche Eigenschaft unserer Sprache ist die Möglichkeit, die Unwahrheit zu sagen, also andere über unsere Gefühle zu täuschen. Die meiste Zeit maskieren wir auf die eine oder andere Weise das, was wir wirklich denken und fühlen"1.

Emotionen äußern sich in der Sprache analog den drei fundamentalen Funktionen des sprachlichen Zeichens, die Karl Bühler in seinem Organonmodell darstellt: Emotionen werden ausgedrückt (Ausdruckfunktion des Zeichens), über Emotionen wird gesprochen (Dar-

1. Heike Ortner, Text und Emotion. Theorie, Methode und Anwendungsbeispiele emotionslinguistischer Textanalyse. Tübingen, Narr, 2014, S. 1. 
stellungsfunktion), und Emotionen werden im Rezipienten erzeugt (Appellfunktion) ${ }^{2}$. Somit beeinflussen sie auch die Art der Gestaltung von Alltagsgesprächen. Sie offenbaren sich u.a. durch spezifische sprachliche Konventionen, die sich mit der Zeit und der wandelnden Gesellschaft verändern. Es ist eine der Aufgaben der Literatur, menschliche und gesellschaftliche Konstellationen auszuprobieren, die es in der Realität so nicht gibt oder sogar gar nicht geben kann. Demgemäß eignet sich der dramatische Dialog als Gegenstand einer gesprächsund emotionslinguistischen Untersuchung. Alltagsgespräche werden im Hinblick auf Regeln und Verfahren untersucht, mit denen die Kommunikationspartner ihre Interaktion praktisch gestalten. Vor allem, wenn soziale Konflikte einer Zeit, aus der keine audiovisuellen Archivierungen vorliegen, sprachwissenschaftlich analysiert werden sollen, bietet es sich geradezu an, literarische Texte heranzuziehen.

Emotionen sind auf allen linguistischen Ebenen des Sprachsystems zu finden ${ }^{3}$. Es gilt nun, herauszufinden, welche sprachlichen Mittel und Formen auf welche Weise Emotionen anzeigen. In einem schriftlichen Text sind das zunächst morpho-syntaktische und lexikalische Mittel. Prosodische Mittel werden hier durch graphische Mittel zumindest annähernd dargestellt, können aber, wie auch non-verbale Mittel, in einem literarischen Text ebenso durch Beschreibungen in der Erzählersprache bzw. in der Figurensprache durch Berichte von Reden anderer Figuren überliefert werden - in einem Dramentext auch durch Regieanweisungen. Es stellt sich auch die Frage nach Kategorisierungen und Konzeptualisierungen von Emotionen, die sich zum Beispiel in Metaphern und Wendungen finden. Natürlich ist in einem Drama die Rolle von Emotionen in Dialogen von großer Bedeutung, da sie auch gesprächsorganisierende Funktionen übernehmen können. Ebenso rücken besondere stilistische Phänomene und emotive rhetorische Mittel in den Fokus.

Im Folgenden werde ich anhand einiger zentralen Szenen aus Hugo von Hofmannsthals Lustspiel Der Unbestechliche $e^{4}$ die Darstellung des Kommunikationsverhaltens der hierarchisch strukturierten Gesellschaft im alten Österreich untersuchen und beschreiben, mit welchen sprachli-

2. Lenka Vaňková, Norbert Richard Wolf, „Literarische Emotionen. Einleitende Hinweise zur emotionslinguistischen Analyse“, in Dies. (Hg.). Aspekte der Emotionslinguistik. Ostrava Facultatis Philosophicae Universitatis Ostraviensis, 2010, S. 7-17.

3. Zu den Fragestellungen der Emotionslinguistik vgl. Heike Ortner, 51f.

4. Hugo von Hofmannsthal, Der Unbestechliche: Lustspiel in fünf Akten. Hrs. Ulrike Landfester. Stuttgart: Reclam, 2000 (=Reclams Universal-Bibliothek, Nr. 18042). URL: http://gutenberg.spiegel.de/buch/der-unbestechliche-999/1 [04.12.2016]. 
chen Mitteln sich die Emotionen in einem heute nicht mehr existierenden sozialen Umfeld manifestieren und wie sie im Gespräch eingesetzt werden, um einen bestimmten Zweck zu erzielen. Das Stück wurde im März 1923 uraufgeführt und spielt laut Personenverzeichnis „auf dem Gut der Baronin in Niederösterreich im Jahre 1912“, also in einem adeligen Haus in der Zeit vor dem Verfall der k.k. Monarchie. Innerhalb dieses Haushalts herrschen Kommunikationspraktiken, welche der traditionellen Etikette entsprechend von einem offiziell-formellen Stil geprägt sind und sich in der Verwendung spezieller sprachlicher Mittel wiederfinden. Die Sprache dient hier zuvörderst der Definition sozialer Verhältnisse; aus dieser Problematik entstehen auch die Konflikte und deren Lösung im Drama. Der Diener Theodor will den Mikrokosmos in diesem Haus nach seinen Vorstellungen organisieren. Der Autor gestaltet also die Kommunikation nach Theodors Intentionen. Er vermittelt somit ein bestimmtes Bild von sozialen Relationen und ihrer sprachlichen Konstruktion im Österreich der späten Monarchie.

Der Unbestechliche wurde als ein für die k.k. Monarchie repräsentativer Text deshalb gewählt, weil hier die Emotionalität ein wesentlicher Teil der Dialoge im Drama sind. Zudem ist dieses Stück auch elektronisch zugänglich ${ }^{5}$, sodass auch Volltextrecherchen möglich sind. Ausgangspunkt aber ist eine diskursive Analyse, d.h. die Analyse erfolgt den Text entlang, und die Funktionen werden aus dem Kontext heraus bestimmt.

So dienen die ersten Szenen des Unbestechlichen der Kontextualisierung des Dramentextes. Das Publikum erlebt zu Beginn ein aufgeregtes Personal, das mit einer Reihe von - derzeit noch unbekannten - Entscheidungen nicht einverstanden ist. Es dreht sich alles um den unmittelbar bevorstehenden Besuch der beiden ehemaligen Geliebten des jungen Barons Jaromir, der verheiratet und Vater von zwei kleinen Kindern ist. Die Hauptperson in diesem Stück ist der unbestechliche Hausdiener Theodor, über den das Publikum bald erfährt, dass er aus zunächst noch nicht bekannten Gründen gekündigt hat und angeblich krank in seinem Bett liegt. Das Stück öffnet mit einer Unmutsäußerung über ihn seitens einer weiteren Dienerin, der Jungfer:

Das ist wieder eine Anordnung vom Theodor. (I, 1)

5. Im Internet-Archiv „Projekt Gutenberg“ liegt eine andere Orthographie vor, z. B. „es wird ja gredt, du bist verliebt in die junge Baronin, deswegen ist dir jetzt unsereins viel zu gewöhnlich!“ $(I V, 4)$. 
Der Zuschauer erfährt gleich zu Beginn, dass es sich um einen Diener handelt, da er nicht mit Nachnamen, geschweige denn Titel benannt wird, der allerdings in der Belegschaft des Hauses eine übergeordnete Rolle innehat, da er seinen Kollegen Anweisungen erteilen kann und dies wohl auch gewöhnlich tut, worauf das Adverb wieder hinweist. Dieses hat hier auch modalisierende Funktion; es offenbart den Unmut der Jungfer über den Sachverhalt. Wieder hat die Bedeutung „aufs Neue, noch einmal“ (Wahrig 2012); damit deutet die Jungfer an, dass Theodor schon öfters Anordnungen gegeben hat, die den Unmut des Personals erregt haben. Ihre Äußerung ist Teil einer nahezu choreographischen Inszenierung der emsigen Betriebsamkeit dreier Bediensteten, in der alle „eilig auftreten“ - und zwar „im größten Tempo“ - und von denen jeder knapp die Situation kommentiert. Die Regieanweisungen haben ebenfalls die Aufgabe, über die Stimmung der Bediensteten zu informieren, die offensichtlich unter großem Druck stehen und dementsprechend erregt sind. Ihre Aussagen wirken zunächst banal, sagen allerdings einiges über das Beziehungsgeflecht unter den Figuren aus. Der zweite „eilige Auftritt“ erfolgt durch die Beschließerin, die nach der „Frau Baronin“ fragt, da sie deren Rat brauche. Während Theodor also Anordnungen erteilt, erteilt seine Dienstherrin lediglich Ratschläge.

Die Äußerung des dritten eilig Auftretenden, nämlich des Gärtners, wendet sich wiederum an Theodor, um sich von diesem neue Anweisungen angesichts einer außerplanmäßigen Situation einzuholen:

GärTnER (eilig auftretend). - Theodor, die Dispositionen müssen geändert werden. Wir haben zu wenig Zimmer. (I, 1)

Die Äußerung des Gärtners ist gewissermaßen eine Reaktion auf die Klage der Jungfer. Er hat, zumindest in dieser Situation die Befehlshierarchie im Hause der Baronin akzeptiert. Deshalb wirkt die vier Mal geäußerte Frage Ist die Frau Baronin nicht da? (I, 1) als Korrektur der Äußerung des Gärtners. Die Frage kommt zum ersten Mal von der Beschließerin, die ja kraft ihres Amtes für die wirtschaftlichen Abläufe in einem adeligen Haushalt verantwortlich ist; sie wiederholt ihre Frage nach dem Weggehen des Gärtners. Der schließt sich bei seinem erneuten Erscheinen der Beschließerin an, und die Jungfer folgt dem Gärtner. Diese Abfolge macht die sozial vorgesehene Hierarchie offenbar, die allerdings im Hause der Baronin keine Geltung haben wird. Die Formulierung der Frage Ist die Frau Baronin nicht da? zeugt einerseits 
vom großen Respekt, den die Bediensteten vor der Hausfrau haben; man könnte durchaus so etwas wie einen 'Bedienstetenjargon' erwarten, etwa Ist die Baronin nicht da?, doch wird sozial vorgeschriebene Form verwendet. Dazu kommt, dass die Frage das Negationszeichen nicht enthält. Negationszeichen kommen dann zum Einsatz, wenn der tatsächliche Sachverhalt anders ist, als der erwartete, der normale. Das will sagen, dass die Frage in dieser Situation, in der sie gestellt wird, keine Äußerung ist, die eine Antwort oder eine Klärung verlangt, sondern die Erstaunen über einen Sachverhalt ausdrückt. Hier scheinen die Figuren nicht ernsthaft zu erwarten, die Dienstherrin als Ansprechpartnerin für die Organisation der Unterbringung der erwarteten Gäste vorzufinden, sondern drücken eher ihren Wunsch aus, sich an sie anstelle von Theodor zu wenden. Die Baronin steht zwar vordergründig im Mittelpunkt des adeligen Haushalts, aber sie ist es nicht, die die Fäden in der Hand hält.

Der Name der Baronin wird an keiner Stelle im Text, nicht einmal im Personenverzeichnis, erwähnt. Ihre Identität beschränkt sich auf ihre soziale Funktion innerhalb der aristokratischen Gesellschaft. An eine ihrer Untergebenen wendet sie sich lediglich mit deren Nachnamen Wallisch, was man heutzutage als eine große Respektlosigkeit interpretieren würde. Durch die namentliche Adressierung gewinnt ihr Ausruf, vor allem mit einer entsprechend eindringlichen Intonation, an Schärfe ${ }^{6}$. Indem sie gar nicht auf den Inhalt der Äußerungen der Beschließerin eingeht, lässt sie die geringe Wertschätzung ihrer Angestellten erkennen. Diese wiederum bezieht sich auf ihren Kollegen mit dessen Vornamen und einem bestimmten Artikel, was auf eine gewisse Vertrautheit bzw. Distanzlosigkeit hinweist. Sie interessiert sich auch nicht für die Klage ihrer Angestellten über Theodor und ihre Rechtfertigungen, und sie lässt diese auch nie zu Ende sprechen, sondern beschließt die zweite Szene nüchtern mit zwei knapp formulierten Ausrufen:

Baronin. - Kein Wort mehr über den Theodor! Genug! (I, 2)

Die abrupte Beendigung des Gesprächs durch die Baronin ist eine Machtprobe; dadurch dass die Baronin so deutlich forciert, will sie ihre eigene "gesteigerte Selbstbestimmung“ sowie die "gesteigerte[...]

6. Vgl. Johannes Schwitalla, „Namen in Gesprächen.“ In: Eichler, Ernst et al. (Hg.), Namenforschung. Ein internationales Handbuch zur Onomastik, Berlin, De Gruyter, 1995. S. 498-504, S. 501. 
Fremdbestimmung" der Beschließerin deutlich machen. Beide Sätze in diesem Gesprächsschritt der Baronin sind elliptisch, wodurch sowohl der Befehlscharakter der Äußerung als auch ihre Verärgerung deutlich zum Ausdruck kommen. Und Genug! am Ende ist die wörtliche Übersetzung von basta! (siehe oben). In der „rhetorischen Grammatik“ bezeichnet man die affektische Spitzenstellung auch als „Ausdrucksstellung“", weil damit die Emotionalität des Sprechers ausgedrückt wird, während die Endposition die „Eindrucksstellung“, ausdrückt das zuletzt Gesagte dem Hörer am besten im Gedächtnis bleibt. Diese beiden Positionen sind hier auf eindrucksvolle Weise genutzt.

Theodor selbst tritt zum ersten Mal in der elften Szene auf. In dem folgenden Dialog mit der Baronin (I, 12) erfährt man endlich gewisse Hintergründe, z.B. warum er gekündigt hat. Zunächst aber will sich die Baronin vergewissern, ob ihr Angestellter tatsächlich beabsichtigt, seinen Dienst zu quittieren. Auf dessen Bestätigung zeigt sie sich überrascht:

BARONIN. - Ja, was soll denn da werden? Sie wissen doch, daß ich zu allem noch Gäste erwarte! (I, 12)

$J a$ ist eine Gliederungspartikel, die grundsätzlich dazu dient, „eine Äußerung einzuleiten, zu gliedern oder abzuschließen" ${ }^{\text {"8. }}$. Hier ist sie in Extraposition und „drückt einen Einwand, eine kritische Bemerkung od. ganz allgemein etwas Negatives, Unerwartetes bzw. Unerwünschtes ${ }^{\text {"9 }}$ aus - und somit die kritische Einstellung der Sprecherin und deren „Distanz von erwartetem und eben bemerkten Sachverhalt" aus, der „auf den Hörer übertragen werden“"10 soll, von dem eine Bestätigung erwartet wird. In der Folge versucht die Baronin, Theodor von dessen Vorhaben abzubringen.

Überhaupt häufen sich in diesem Gesprächsschritt die Modalpartikeln. Denn ist hier ein Hinweis, dass die Baronin Theodor einen Vorwurf macht. Sie erwartet somit keine Antwort auf ihre Frage, sondern

7. Vgl. Werner Kallmeyer, Reinhold Schmitt. „Forcieren oder: Die verschärfte Gangart. Zur Analyse von Kooperationsformen im Gespräch“. In: Werner Kallmeyer (Hg.). Gesprächsrhetorik: Rhetorische Verfahren im Gesprächsprozess. Tübingen, Narr, 1996. S. 19-118, S. 22.

8. Métrich, René, Faucher, Eugène. Wörterbuch deutscher Partikeln. Unter Berücksichtigung ihrer französischen Äquivalente. Berlin, Walter de Gruyter, 2009, S. 519.

9. Ebd.

10. Gerhard Helbig. Lexikon deutscher Partikeln. Leipzig: Langenscheidt Verlag Enzyklopädie, ${ }^{3} 1994$, S. 167. 
eine Rechtfertigung ${ }^{11}$. Durch den Gebrauch von doch will sie daran erinnern, dass es Theodor klar ist, in welche missliche Lage er sie durch seine Kündigung bringen würde. Zumindest signalisiert sie, dass sie davon ausgeht. Sie appelliert an ihr gemeinsames Wissen und an sein Verantwortungsbewusstsein ${ }^{12}$. Auch noch ein Indiz für ihre Erregung. In diesem Fall liegt keine zeitliche Bedeutung vor, sondern es wird nervliche Anspannung bekundet. Die Baronin verweist damit auf die zusätzliche Komplikation, nämlich dass eine Invasion von Gästen unmittelbar bevorsteht und gleichzeitig erzeugt sie einen „drohende[n] Unterton]“ in Bezug auf die „zu erwartende[n] Konsequenzen“ von Theodors potentiellem Handeln ${ }^{13}$.

Allerdings gilt diese Drohung lediglich seinem Gewissen, denn die Baronin steht machtlos der Tatsache gegenüber, dass er sich aus seinem Dienst entfernen könnte. Dies ist für ihn als gesellschaftlich Unterlegenen das einzige Mittel, dass ihm zur Verfügung steht, um seinen Willen gegenüber seiner adeligen Dienstherrin durchzusetzen. Er wendet es als Erpressungsmethode, da sich die Baronin offensichtlich von seinen Diensten abhängig wähnt. Da er nun aber aufgrund seiner sozialen Stellung ebenso von ihr abhängig ist, gibt er sich in seinem Verhalten und seiner Rede stets unterwürfig, wenn er auch jegliche Verantwortung von sich weist:

THEODOR (mit bedauernder Gebärde). - Es ist mir selber sehr peinlich, aber sehr gewichtige Umstände haben mich in die Zwangslage versetzt - $(1,12)$

Die Baronin unterbricht ihren Diener, was wiederum eine typisch forcierende Aktivität in der sprachlichen Interaktion darstellt, die sie sich in ihrer Position erlauben kann. So verstärkt sie ihre eigenen Rechte und gleichzeitig die Pflichten des Gesprächspartners, aber in ihrer Antwort spricht sie ihn im Gegensatz zu seinen Kollegen mit seinem Vornamen an. Dies belegt, dass zwischen den beiden trotz der sozialen Unterschiede die Distanz geringer ist. Er wiederum spricht sie, wie alle anderen Dienstboten, mit der Anrede im Plural an und reagiert mit einer Unterlegenheitsgeste, statt auf ihre Frage zu antworten:

Theodor. - Euer Gnaden bitte ich nur in untertänigster Dankbarkeit die Hände küssen zu dürfen. (I, 12)

11. Ebd., S. 106.

12. Vgl. Ebd., S. $111 \mathrm{ff}$.

13. http://www.duden.de/rechtschreibung/noch_schon_mal_doch\#Bedeutung3

[28.04.2017]. 
Theodor führt seine Strategie der Nonresponsivität ${ }^{14}$ kombiniert mit Demutsgebärden fort und bringt so auf geschickte Weise die Baronin, die nie die von ihr erwartete Reaktion erhält, ihn dazu, aufzufordern, seine persönliche Meinung über familieninterne Angelegenheiten seiner Dienstherren zu äußern, was ihm unter normalen Umständen nie zustehen würde. Dabei bleibt er zunächst bei sehr allgemeinen Aussagen, redet um den heißen Brei herum und spielt zunächst nur vorsichtig auf die lasterhafte Lebensführung des jungen Barons Jaromir an:

TheODOR. - Der Herr Oberst hat mir in seiner letzten Lebensstunde gesagt, daß ich ihm meine Jugend aufgeopfert habe, und hat mich mit Tränen in seinen sterbenden Armen beschworen, seinen Jaromir nicht im Stich zu lassen und mir den heiligen Eid abverlangt, daß ich dem jungen Herrn mein Mannesalter aufopfern werde. Denn er hat die vielen und großen Schwächen dieses Jünglings erkannt. (I, 12)

Er versteckt seine Kritik also hinter den letzten Worten des alten Barons, worauf dessen Gattin natürlich nicht widersprechen kann, sondern Theodor sogar lobt:

BARONIN. - Und dann haben Sie siebzehn Jahr im Dienst meines Sohnes verbracht und sich tadellos geführt. Aber endlich haben gewisse Verschiedenheiten in Ihren beiden Charakteren es wünschenswert erscheinen lassen, daß Sie aus seinem Dienst wieder in meinen traten, was mir natürlich sehr lieb war. (I, 12)

Hier setzt Theodor nun mit einer offeneren Darstellung seiner Gefühle an:

Theodor. - Das könnte man gesellschaftlich so sagen, aber es wäre weiter nichts als eine vertuschende Redeweise.

Sehr stark, aber nicht laut.

Die Wahrheit ist diese: das ganze Leben, das er geführt hat, war eine fortgesetzte Beleidigung meiner Person. (I, 12)

Dabei gibt er sich sichtlich und hörbar erregt, denn wir man aus der Bühnenanweisung erfährt, hebt er seine Stimme, aber ohne wirklich laut zu werden, was vermuten lässt, dass er die Stimm-Modulation aus strategischen Gründen bewusst einsetzt. Explizit drückt er sich dennoch noch nicht aus, aber die Baronin versteht offenbar, worauf er anspielt:

BARONIN. - Pst, pst. Sie sprechen von meinem Sohn! (I, 12)

14. Vgl. Robert Mroczynski, Gesprächslinguistik. Eine Einführung. Tübingen, Narr, 2014, S. 76. 
Es ist nicht eindeutig, ob sie ihn zum Schweigen bringen will oder ihn veranlassen möchte, leiser zu sprechen. Im Gegensatz zu den anderen Bediensteten wie dem Gärtner und der Beschließerin erteilt sie ihm keinen Befehl, sondern drückt durch den Exklamativsatz eine Ermahnung oder eine Befürchtung aus. Das Ausrufezeichen weist darauf hin, dass es sich hier nicht um eine neutrale, konstative Aussage handelt. Zum einen erinnert die Sprecherin daran, dass Theodor sowohl eine persönliche als auch eine gesellschaftliche Grenze überschreitet, indem er Kritik an ihrem Sohn übt. Zudem befürchtet sie wohl auch, dass andere Mitglieder des Haushalts diese Transgression mitbekommen oder etwas Indiskretes über ihren Sohn erfahren. Je nach Auslegung der Rolle und der Intention der Sprecherin ist Sohn oder das entsprechende Possessivpronomen zu betonen. Auf jeden Fall handelt es sich hier um einen performativen Sprechakt, mit dem sie sie auch dieser Stelle an Theodors Gewissen appelliert, der daher das Gelingen seines Sprechakts gefährdet sieht und zum zweiten Mal mit der typisch österreichischen Demutsgebärde reagiert:

THEODOR stehend. - Ich bitte nichts anderes, als die Hände küssen und mich stillschweigend untertänigst zurückziehen zu dürfen, auf immer. (Als wollte er gehen.) (I, 12)

Die Bühnenanweisung belegt, dass er gar nicht die Absicht hat, zu gehen. Vielmehr bringt er die durch seine manipulative Rede die Baronin dazu, ihn geradezu zu zwingen, sich offen auszudrücken. Diese reagiert auf seine Aussagen auch tatsächlich völlig anders als auf die seiner Kollegen und spricht ihm wiederum beim Vornamen an:

BARonin. - Ich wünsche aber, daß Sie bleiben, Theodor. (I, 12)

Die namentliche Referenz dient dazu, dem Wunsch einen besonderen Nachdruck zu verleihen und hat hier beziehungsintensivierende Funktion. Somit wird dem Appell ein gewisser emotionaler Gehalt verliehen, mit dem Theodor moralisch verpflichtet werden soll, auf seiner Stelle zu bleiben.

Theodor betont in einer überaus emotionalen Rede, dass er großen Wert darauf lege, mit seinem richtigen Namen gerufen zu werden:

Theodor. - Jawohl, meine Eltern haben mir in der heiligen Taufe den lieben Namen Theodor zugeeignet. Er hat den Namen nicht beliebt. Ich bin bei ihm die Jahre hindurch Franz gerufen worden, Franz, wo ich, bitte, Theodor zu heißen die Ehre habe! Darin bitte zu erkennen, wie er die Menschenwürde in mir geachtet hat! Das Ganze war eine siebzehnjährige automatische Mißachtung. (I, 12) 
Seinen Ärger darüber, anders genannt zu werden, bringt er mit Ironie zum Ausdruck, indem er von der Achtung der Menschenwürde spricht, die Jaromir ihm zuteilwerden ließ. Er meint damit natürlich genau das Gegenteil. Mit dieser Art der Äußerung erzielt er auch eine andere Wirkung, als wenn er schlicht sagte: „Ihr Sohn hat meine Menschenwürde nicht geachtet.“ Denn: „Je stärker ironische Bemerkungen vom eigentlich Gemeinten und vom Erwartbaren abweichen, desto expressiver und emotionalisierender werden sie beurteilt. ${ }^{{ }^{15}}$ Gleichzeitig dient die Ironie der Gesichtswahrung, weil die Kritik nicht direkt formuliert wird. Die Baronin hat hier also keine Gelegenheit, ihm zu widersprechen.

Theodor hat - wie wir in der vierten Szene erfahren haben - seine Stellung bei der Baronin aus noch unbekannten Gründen gekündigt. In diesem Gespräch äußert sie den Wunsch, dass er bleibt. In seiner Antwort verwendet Theodor zunächst die verstärkte Responsivpartikel ,jawohl“, die allerdings keine Antwort auf eine Entscheidungsfrage ist, sondern auf den Gesprächsschritt des Dieners vor dem Wunsch der Baronin verweist und dort anknüpft. Wiederum hat die Responsivpartikel die Funktion eines Konnektors, der zudem einen Gegensatz zur Äußerung der Baronin andeutet. Der Autor hat zwischen der Responsivpartikel und dem nachfolgenden Text ein Komma gesetzt, was deutlich macht, dass Theodor mit ,jawohl“ nicht zusagt, dem Wunsch der Baronin nachzukommen. Er schließt vielmehr an die frühere Äußerung an:

Die Wahrheit ist diese: das ganze Leben, das er [i.e. Jaromir, der Sohn der Gräfin] geführt hat, war eine fortgesetzte Beleidigung meiner Person. (I, 12)

Warum Theodor von einer Beleidigung spricht, erläutert der folgende Text. Seine Erregung ist so stark, dass er auf die Reden der Baronin gar nicht eingeht, durch die Partikel jawohl wird die starke emotionale Beteiligung Theodors deutlich. Dazu kommen die attributiven Adjektive heilig und lieb, die signalisieren wie ernst der Diener das Fehlverhalten des Sohnes Jaromir nimmt. Jaromir hat keinen Respekt vor dem Sakrament der Taufe und dem Namen des Dieners, denn dieser lieb gewonnen hat, woraus eine starke Verärgerung Theodors resultiert und er eine Missachtung seiner Menschenwürde sieht. Sowohl der Satz, in dem Theodor kund tut, dass Jaromir ihn mit Franz ruft, als auch der Satz, in dem es um seine verachtete Menschenwürde geht, sind mit Ausrufezeichen abgeschlossen, die deutlich machen, wie wichtig Theodor diese ganze Angelegenheit ist.

15. Heike Ortner, Text und Emotion, S. 276. 
Die Verärgerung Theodors wird in dem abschließenden Satz „Das Ganze war eine siebzehnjährige automatische Mißachtung“ zusammengefasst. Theodor verwendet eine Reihe von sehr feierlich klingenden Substantiven und Redewendungen („die Ehre haben“), die deutlich die Emotionalität dieses Redeschritts formulieren.

Die Baronin erwidert, dass die von Theodor vorgebrachte Beschwerde von geringer Bedeutung ist:

BARonin. - Aber das sind doch schließlich nur Kleinigkeiten. (I, 12)

Die Modalpartikel doch signalisiert hier eine überraschende Feststellung der Baronin, die ihrer Erwartung widerspricht ${ }^{16}$. Verstärkt wird dies durch die Kombination mit der Modalpartikel schließlich, mit der die Sprecherin ihre Einstellung wiedergibt, dass die Tatsache, dass es sich um „Kleinigkeiten“ handle, außer Frage stehe, was man "nicht unberücksichtigt lassen darf, da dieser Umstand alles erklärt. ${ }^{17}$ Gleichzeitig fordert die Baronin also ihren Gesprächspartner damit zur Zustimmung auf, die sie aber nicht erhält:

Theodor. - Kleinigkeiten? Für die menschliche Seele gibt es keine Kleinigkeiten, das müssen Eurer Gnade als hochgeborene und gebildete Dame wissen. Er hat vor meinen sehenden Augen ein Junggesellenleben geführt von einer beispiellosen Frivolität und eiskalten Selbstsucht. (I, 12)

Mit der als Frage formulierten Wiederholung von Kleinigkeiten will sich Theodor nicht ernsthaft vergewissern, dass er richtig gehört hat, sondern signalisiert seinen Widerspruch. Er wird in seinen Anschuldigungen gegenüber Jaromir nun explizit und verwendet dabei die wertenden und somit emotiv getönten Adjektive beispiellos und eiskalt, entschärft aber seine Rede, indem er sie als hochgeboren und gebildet bezeichnet. Somit verhindert er eine Verschlechterung des Gesprächsklimas und präsupponiert gleichzeitig, dass die Baronin wider besseren Wissens womöglich nur aus dem Affekt heraus gesprochen hat. Da sie weder dies bestätigen will noch als ungebildet gelten oder ihren sozialen Rang in Frage stellen will, bleibt ihr nichts als zu schweigen. Ihren Unmut bringt sie laut Bühnenanweisung nonverbal zum Ausdruck:

Baronin stößt mit dem Stock. (I, 12)

16. Vgl. Gerhard Helbig, Lexikon deutscher Partikeln, S. 116.

17. Ebd., S. 199. 
Obwohl sie damit nichts als ihre emotionale Befindlichkeit kommuniziert, pflichtet Theodor ihr bei:

Theodor. Sehr richtig! Sie klopfen, Sie haben recht! (I, 12)

Auch dagegen kann die Baronin nichts einwenden und Theodor kann seine Anklage fortfahren:

Ich habe es ertragen. Ich habe Krawatte hergerichtet, den Jackett oder Smoking, wenn ich gewußt habe, er geht darauf aus, ein weibliches Wesen in einer nächtlichen Abendstunde mit kaltherziger Niederträchtigkeit um die Seele zu betrügen. $(I, 12)$

Die xenolektalen Phänomene wie ein fehlender Artikel, ein falsches Genus oder eine Abweichung von der Regelsyntax ${ }^{18}$ zeigen, dass der sonst so eloquente Böhme, der sich grundsätzlich an die standardsprachlichen Normen des Deutschen hält und diese auch beherrscht, nicht nur künstlich aufgeregt ist, während bis jetzt nicht eindeutig war, ob er diese Emotion nicht als rhetorisches Mittel einsetzt und theatralisch inszeniert. Jedenfalls wurde es als typisch österreichisch angesehen, dass in einem wohlhabenden Haushalt unter anderem auch Personal beschäftigt ist, das durch den Akzent sofort als böhmischstämmig identifiziert werden kann. Beabsichtigt oder nicht, Theodors Vorgehen hat Erfolg, denn er hat längst die Führungsrolle in diesem Gespräch übernommen. Seine Vorgesetzte gibt nur noch knappe Äußerungen von sich und akzeptiert es sogar zweimal, von ihm unterbrochen zu werden. Sie widerspricht ihm nicht, sondern versucht, zu beschwichtigen:

BAronin. - Aber Theodor, Sie sind mir doch auch kein Heiliger! (I, 12)

In diesem Fall signalisiert die Modalpartikel doch eine spontane Reaktion auf die eben gewonnene Erkenntnis der Baronin, die offensichtlich nicht erwartet hat, dass der wohl allgemein bekannte - und gesellschaftlich tolerierte - Lebensstil ihres Sohnes der Grund für Theodors Kündigung ist. Sie erwartet durch ihre Äußerung seine Zustimmung zu der Tatsache, dass sein eigener Lebenswandel dem seines ehemaligen Vorgesetzten in einer gewissen Weise ähnelt. Theodor rechtfertigt sich, in dem er sich auf den Einsatz seines aufrichtigen Gefühls bei seinen erotischen Unternehmungen beruft, dass bei Jaromir seines Erachtens fehle:

18. Das Tschechische, das in diesem Fall die Substratsprache ist, hat keine Artikel. Zudem hat Jackett hier einen falschen, weil tschechischen Artikel. 
Theodor. - [...] Aber wenn ich eine liebende Handlung begehe, so begehe ich sie mit meinem ganzen Herzen und stehe dafür ein mit meiner ganzen Seele. Bei ihm ist aber das Gegenteil der Fall, und das kann ich nicht mehr vertragen mit meinem Auge zu sehen! (I, 12)

Eine xenolektale Wortfolge wie im letzten Satz kommt bei Theodor typischerweise in einer besonders emotionalen Situation vor.

Als er schließlich auf „einen sogenannten Schlüsselroman ohne einen literarischen Wert" zu sprechen kommt, in dem der Autor Jaromir seine Liebesabenteuer festgehalten hat, greift die Baronin zu einem völlig neuen Mittel, das Thema zu einem abrupten Abschluss zu bringen:

BARONIN. - Ich verstehe absolut nicht, wovon Sie reden, Theodor. (I, 12)

Man kann davon ausgehen, dass dir Baronin nichts davon wissen will, da zum einen das Metier des Schriftstellers nicht unbedingt dem Stand des Adels entspricht und weil sie zum anderen womöglich darüber informiert ist, dass das literarische Werk ihres Sohnes ,große Diskretionssachen", wie es Theodor in einem späteren Gespräch mit Melanie Galattis ausdrückt, enthält. Um das Gespräch dennoch fortführen und sein Anliegen weiter darlegen zu können, bietet er nun wiederum mit Erfolg seinen strategischen händeküssenden Rückzug an.

In der Folge drückt er sich viel direkter aus und setzt sogar zu längeren Reden an, welche die Baronin nur noch klopfend bzw. mit kurzen Exklamationen kommentiert. Dies ist umso bemerkenswerter, als ein derartiges Ungleichwicht in einem Dialog typischerweise zugunsten des sozial höhergestellten Sprechers auswirkt: „Das Auftreten von Assymetrien auf lokaler Ebene ist stark mit dem Sprecher- und Hörerstatus der Gesprächsteilnehmer verknüpft, der schon an sich eine asymmetrische Erscheinung darstellt. ${ }^{19}$ Theodor ergreift wesentlich länger als seine Dienstherrin das Wort und schließlich fordert sie ihn sogar mit einer ihre Verzweiflung signalisierenden Demutsmetapher zum Sprechen auf:

BAronin. - An was denken Sie denn? So reden Sie doch! Ich bitte Sie mit aufgehobenen Händen - so reden Sie doch! (I, 12)

Noch leistet die Baronin Widerstand, als Theodor seine Absicht verkündet, dafür zu sorgen, dass die beiden ehemaligen Geliebten, die gerade am Gut der Familie eintreffen, baldmöglichst wieder abreisen:

19. Robert Mroczynski, Gesprächslinguistik, S. 214. 
BARonin. - Ja, wie denn, um Gottes willen? Sie werden doch nicht in offener Opposition meinem Sohn entgegentreten wollen? (I, 12)

Mit dem Emotionsausdruck um Gottes willen kommuniziert sie ihr Entsetzen über die Vorstellung, dass ein Dienstbote die Autorität ihres Sohnes untergraben könnte, allerdings signalisiert sie sogleich, dass sie bereit wäre, entgegen ihrer Überzeugungen zu handeln, wenn Theodor im Gegenzug in seinem Dienst bleiben würde:

BARONIN. - Und eine solche Lösung wäre, wenn sie denkbar wäre, - würden Sie - Sie würden dann Ihre Kündigung zurücknehmen? $(\mathrm{I}, 12)$

Dennoch zeigt die Tatsache, dass sie ihren Satz abbricht und neu ansetzt, dass sie gefühlsmäßig verwirrt ist. Es handelt sich hier nicht um eine bewusst eingesetzte Aposiopese der Sprecherin, sondern um eine Retraktion, d.h. ein Abbruch mit anschließender Korrektur ${ }^{20}$. Solche Abbrüche entstehen häufig durch Ablenkung oder schnelles Sprechen, was beides auf emotionale Erregung hinweist. Theodor antwortet ausweichend, stellt aber eine Entscheidung im Sinne seiner Vorgesetzten in Aussicht, welche, als sie erfährt, dass die Gäste soeben eingetroffen sind, wieder in ihre Rolle der sozial Dominierenden zurückkehrt und die Szene mit einem Befehl beendet:

BARonin. - Bleiben Sie hier! (I, 12)

In der übernächsten Szene führen die beiden ihr Gespräch fort. Die Baronin hat sich mittlerweile gefasst und setzt zu einem längeren Diskurs an, mit dem sie Theodors Bedingungen für ein Verbleiben offenbar neu verhandeln will. Ihre emotionale Verwirrung offenbart sich dabei in einer Wärme-Konzeptualisierung:

BAronin. - Also kommen Sie her, Theodor. Schnell. Sie haben mir da früher Dinge vorerzählt, ich habe einen ganz heißen Kopf bekommen. Ich hab nur so viel daraus entnommen, daß Sie unter gewissen Bedingungen, von denen ich allerdings nicht ahne, wie sie könnten erfüllt werden, bleiben würden. Ich kann nur eines sagen... (I, 14)

Theodor lässt sich aber auf keinen Kompromiss ein und unterbricht sie wieder. Er verliert immer mehr die Fassung, wie es unter anderem die xenolekte Syntax nochmals widerspiegelt:

20. Ebd., S. 162. 
THEODOR. - Ich glaube von meinen Bedingungen in deutlicher Weise gesprochen $\mathrm{zu}$ haben. Meine Genugtuung wünsche ich zu erblicken darin, daß das ganze Gebäude von Eitelkeit und Lüge zusammenstürzen muß, als eine unbegreifliche Wirkung meiner höheren Kräfte. (I, 14)

Er beherrscht jetzt den Diskurs und übt so Macht über die Baronin aus: Er kann es sich erlauben, das Wort zu ergreifen, wann er möchte, er ist derjenige, der die Bedingungen stellt und er kommuniziert jetzt offen, dass es ihm um seine persönliche Genugtuung geht. Seine Beurteilung von Jaromirs Lebensstil kommuniziert er eindringlich mit einer Architektur-Metapher, die das drastische Ausmaß seiner Laster verdeutlicht und er schätzt seine eigenen Fähigkeiten als höhere Kräfte ein. Er nimmt seinen sprechenden Namen, auf den er so besteht, also offenbar wortwörtlich: „Gottes Geschenk ${ }^{\text {“21. }}$.

Allerdings wehrt sich die Baronin immer noch gegen die Durchsetzung seiner Forderungen und setzt wieder mit vorgespieltem Unverständnis zu einem Ausweichmanöver an:

BARonin. - Ich habe keine Ahnung, was Sie mir da vorgeredet haben. (I, 14)

Theodor durchschaut ihre rhetorische Strategie und wirft ihr genau das vor, was er selbst schon mehrfach getan hat und nun erneut tut: Er zieht sich wiederum aus der Affäre, wenn auch hier zum ersten Mal ohne verbalen Handkuss, da er dies wohl nicht mehr nötig hat. Stattdessen greift er zu einer Ausrede in Form einer sexistischen Stellungnahme:

Theodor. - Mir ist diese ausweichende Redeweise bei weiblichen Personen bekannt. Demgemäß werde ich mich in Ruhestand zurückziehen. (Er heftet einen durchdringenden Blick auf sie). (I, 14)

Die beiden liefern sich keinen offenen rhetorischen Schlagabtausch, sondern agieren taktisch in ihren traditionellen sozialen und geschlechtlichen Rollen, wobei ihr Dialog dennoch nichts als ein Tauziehen um Einflussnahme über den Gesprächspartner ist. Theodor setzt hier seine xenolektale Syntax möglicherweise bewusst ein, um sich mit seiner vermeintlich mangelnden Sprachkompetenz auf seiner gesellschaftlich niedrigeren Stufe zu positionieren, begibt er sich rhetorisch

21. Hofmannsthal selbst äußerte, dass Theodor sich "für etwas Christus Verwandtes“ halte (zitiert nach Ulrike Landfester. „Nachwort“. In: Hugo von Hofmannsthal. Der Unbestechliche. Stuttgart: Reclam, 2000 [=Reclams Universal-Bibliothek, Nr. 7828], S. 101). 
auf strategischen Rückzug, in dem er sagt, dass er sich in Ruhestand zurückziehen will. Seine nonverbale Kommunikation, von der wir durch die Bühnenanweisung erfahren, widerspricht seiner Äußerung. Der Baronin eine ausweichende Redeweise vorwerfend, kündigt er sein eigenes Ausweichen an, signalisiert mit seiner Mimik aber eher, dass er eine Reaktion von ihr erwartet. Die Baronin erwidert daraufhin, laut Bühnenanweisung, schnell. Die Steigerung der Sprechgeschwindigkeit ist, wie bereits erwähnt, ein Hinweis auf emotionale Erregung, und es ist anzunehmen, dass der starre Blick ihres Angestellten sie in Verlegenheit bringt. Mit ihrer Antwort weicht sie tatsächlich wieder aus und erklärt keineswegs, dass sie Theodors Bedingungen akzeptiert, sondern lediglich, dass sie ihn im Dienst behalten möchte. Er ignoriert dies aber und sieht seine Forderungen offensichtlich erfüllt. In der Folge erfahren wir, dass er längst seine Manipulationsmanöver geplant und in die Wege geleitet hat. Den Umgang mit Frauen geht er an wie ein Billardspiel, wie er selbst angibt: Die eine der beiden von Jaromirs Ex-Geliebten, Melanie Galattis will er von der Bande anspielen, da sie wie die meisten Frauenzimmer dumm und gescheit zugleich sei. Paradoxerweise hat er, um die verflossenen Mätressen aus Gründen der Moral so bald wie möglich aus dem Schloss zu entfernen, seine eigene ehemalige Liebhaberin, von der er sich aus unbekannten Anlass getrennt hatte, bereits kommen lassen, damit sie ihn ohne ihr unbekanntem Wissen bei seinen Machenschaften unterstützt. Er ist sich ihrer Dienstbarkeit auch sicher:

Theodor. - Ich habe ihr verziehen und dies in einem Brief zu erkennen geben. Sie wird demgemäß heute abend glücklich erscheinen und mir blind ergeben sein. (I, 14)

Seitens der Baronin gibt es keine Einwände mehr, geschweige denn Widerspruch. Ganz im Gegenteil erfragt sie sogar Anordnung für ihr eigenes Verhalten in der Intrige:

BARonin. - Meinetwegen. Und was soll ich tun? (I, 14)

Allerdings widerstrebt es ihr immer noch, Theodors Forderung nachzukommen, nämlich ihm in diskreter Weise freie Hand zu lassen, wie er es verlangt, und flüchtet sich wieder in ihre gefühlsmäßige Verwirrtheit:

BARonin. - Ich beschwöre Sie, Theodor, ich weiß ja nicht, wo mir der Kopf steht.

Theodor. - Ich bitte, jetzt keine Beschwörungen mehr anzuwenden, sondern lediglich ein einziges Wort auszusprechen, damit jedermann in diesem Haus weiß, woran er sich zu halten hat. (I, 14) 
Der Diener zeigt somit, dass er seine Autorität über die Kollegen nur behalten kann, wenn diese von der obersten Instanz im Hause diktiert wird. Ohne das Machtwort der Baronin ist er selbst unter den Dienstboten machtlos. Daher zieht er alle Register, um im Dialog mit der Dienstherrin die Oberhand zu gewinnen, wobei er seinem Ziel immer näher kommt:

BARONIN. - Ich sprech gar nichts. Ich will gar nichts wissen. Was für ein Wort denn?

Theodor. - Euer Gnaden werden ganz einfach sagen: „Und Sie, lieber Theodor, übernehmen jetzt wieder die Aufsicht über das Ganze.“ Dies bitte ich auszusprechen, wenn das niedrige Personal gegenwärtig sein wird. (I, 14)

Theodor passt sich hier zumindest äußerlich wieder den gesellschaftlichen Gesprächsnormen an. Seine Vorgesetzte gibt schließlich nach, was sich schon dadurch andeutet, dass sie sich nach der Dienstanweisung erkundigt, die Theodor von ihr erwartet, und zwar unmittelbar nachdem sie verkündet hat, sie gar nicht wissen zu wollen. Ihr Widerwille äußert sich dabei in einer Sprechweise, die sie bis jetzt nicht an den Tag gelegt hat: Die Flexion $-e$ der Verben in der ersten Person Singular spricht sie mehrmals hintereinander nicht aus, was generell charakteristisch für mündliche Umgangssprache ist, womit deutlich wird, dass sie ihre Position aufgegeben hat. Allerdings weist sie noch darauf hin, dass sie ihm überhaupt kein Zugeständnis gemacht hat:

BARONIN. - Aber ich hab doch gar nichts mit Ihnen verabredet!

Theodor. - Sehr wohl. Darauf werde ich bestehen, daß es wörtlich ausgesprochen wird und in einer äußerst huldvollen Weise: „Und Sie, lieber Theodor, übernehmen jetzt wieder die Aufsicht über das Ganze.“ Es wird für mich eine geheime unterirdische Bedeutung haben, die anzuhören meinen Ohren eine schmeichelhafte Genugtuung bereiten wird. (Sieht sie scharf an.)

BARonin. - Also, ich werd es sagen, ich werd es sagen - $(\mathrm{I}, 14)$

Die Baronin soll Theodor auf eine äußerst huldvolle Weise ansprechen, damit seine Autorität über die untergeordneten Diener wieder hergestellt wird. Mit der Responsivpartikel also resümiert sie den Ausgang des Gesprächs: Sie hat seine Forderung zur Kenntnis genommen, wird nun entsprechend reagieren und signalisiert gleichzeitig das Ende des Gesprächs. Indem sie ihre Zusage widerholt, intensiviert sie diese, was einem Versprechen gleich kommt - Theodor hat sein Ziel erreicht. Das ganze Manöver dient also auch zur Wiederherstellung seiner Macht im Kollegium, und schließlich übernimmt er auch das Regiment im Haus. 
Theodor mag zwar gesellschaftlich der Baronin weit unterlegen sein, aber im Gespräch war er eindeutig der Dominante. So hat er die sprachliche Handlung erfolgreich vollzogen und seinen Willen durchgesetzt.

Gesondert behandelt gehören die Diminutiva mit den Suffixen -1 und -erl wie in Büchel, die als typisch ostoberdeutsch und hier besonders österreichisch gelten. Sie werden häufig in emotionaler Funktion in österreichischer „Oberschichtensprache“ verwendet, daneben auch mit hypokoristischen Aufgaben in Reden mit Kindern wie dem kleinen Jaromir.

Theodors Diskurs ist im Umgang in der Gesellschaft von den sprachlichen Konventionen des österreichischen Kleinadels und dessen Dienstpersonal geprägt, an die er sich offensichtlich hält. Es handelt sich insofern um eine unsymmetrische Kommunikation mit allen Kennzeichen (Anreden, Antworten), der österreichischen Adelskommunikation in der späten Monarchie: Der Baronin antwortet Theodor stets bejahend (Sehr richtig! Sie klopfen, Sie haben recht!, I, 12) oder ausweichend, wobei er sich an österreichische sprachliche Gepflogenheiten eines sozial Untergebenen hält: „Ich bitte nichts anderes, als die Hände küssen und mich stillschweigend untertänigst zurückziehen zu dürfen“" (ebd.). Hier handelt es sich nicht etwa um eine knappe Bestätigung, sondern um (scheinbare) Demutsgesten (Hand küssen). Statt Widerspruch von unten nach oben gibt es non-responsive Antworten. Allerdings deutet schon die wiederholte Regieanweisung Als wollte er gehen darauf hin, dass Theodor nichts anderes vorhat, als seine Meinung deutlich zu sagen und "die Aufsicht über das Ganze" zu fordern, was ihm die Baronin schließlich gewährt. Geschickt steuert er so sein Nähe-Distanz-Verhalten. Seine erfolgreiche Strategie besteht in der Durchführung emotiver sprachlicher Handlungen, indem er echte oder vorgetäuschte Gefühle zum Ausdruck bringt und in der Baronin erzeugt. Mit der Drohung, aus seinem Dienst auszuscheiden, erzeugt er ihre Angst, was schließlich zum Gelingen seines Sprechaktes führt. Die Szenen I, 11 und I, 14 wurden als Mittelpunkt dieser Recherche ausgewählt, weil hier mit großem emotionalem Einsatz die sozialen Rollen auf dem Gut von österreichischen Landadeligen und ihren Bediensteten mit sprachlichen Mitteln festgelegt werden. Repräsentanten der über- bzw. untergeordneten Gesellschaftsschicht korrespondieren miteinander (in den Szenen wird auch Theodors Herkunft angesprochen) und die Auseinandersetzung ist stark emotionalisiert und gleichzeitig kodiert.

Nachdem Theodor die Wiederherstellung der Ordnung vollendet hat - worum er gar nicht gebeten wurde - zieht er sich selbst diskret 
zurück, allerdings nicht auf sein Zimmer, sondern er macht sich nun seinerseits zu einem Stelldichein mit der Dienerin Hermine. Wenn hier also so etwas wie eine Revolution stattfindet, dann besteht sie darin, sich die Privilegien des Adels anzueignen und dessen Lebensstil zu imitieren. Das Spiel um Verrat, Lüge und Moral setzt sich folglich fort und steht beispielhaft für die Gesellschaft in der untergehenden habsburgischen Monarchie. Auf diese Weise dienen die Dialoge der Beschreibung gesellschaftlicher Verhältnisse; Gesellschaftskritik ist somit Sprachkritik.

Mit anderen Worten: Hofmannsthal präsentiert eine Gesprächsform, wie er sie für die österreichische Landadelsgesellschaft für typisch gehalten hat. Kennzeichnend ist auch die Tatsache, dass ein Mitglied des Dienstpersonals aus Böhmen stammt, das an Niederösterreich angrenzt. Bis zum Ende der Habsburger Monarchie, waren Hausangestellte aus anderen Kronländern bei adeligen und bürgerlichen Familien sehr häufig. In der Aufführungspraxis dieses Stücks ist es auch seit Beginn Tradition, dass der Darsteller Theodors „böhmelt“. Robert Musil notierte über die Uraufführung: „Pallenberg gab ihm einen leisen wie von vielen in deutscher Umgebung verbrachten Jahren verschlissenen tschechischen Akzent“ ${ }^{\text {‘22 }}$. Ein vergleichbares Stück scheint es außerhalb Österreichs nicht zu geben. Dabei ist von Bedeutung, dass sich Theodor fast immer im Rahmen dieser Gesprächsformen bewegt; nur in Dialogen wie mit dem kleinen Jaromir wird er ein ganz Anderer. „Österreichisch“ heißt also nicht nur, sogenannte Austriazismen, also lexikalische Elemente zu benutzen, sondern sich bestimmter Diskursformen zu bedienen. Freilich ist dies ein literarisches, will sagen: fiktionales Konzept, dessen Realitätsgehalt deshalb nicht überprüft zu werden braucht.

22. Robert Musil, „Der Unbestechliche v H v H (Uraufführung im WR R TH) [Mitte März 1923]." In: Adolf Frisé (Hrsg.) Robert Musil. Gesammelte Werke: 2. Essays und Reden. Kritik. Hamburg: Rowohl, 1978, S. 1731. 